\title{
RDUS
}

Revue de DROIT

UNIVERSITÉ DE SHERBROOKE

\author{
AFFAIRE DE LA NOMINATION DU JUGE MAINVILLE À LA COUR \\ Titre : D'APPEL DU QUÉBEC : UNE CONCESSION ET TROIS CRITIQUES
}

Auteur(s): Maxime ST-HILAIRE

Revue : $\quad$ RDUS, 2015, volume 45, numéro 3

Pages: $\quad 575-596$

ISSN : $\quad$ 0317-9656

Éditeur : $\quad$ Université de Sherbrooke. Faculté de droit.

URI : $\quad$ http://hdl.handle.net/11143/9921

DOI : $\quad$ http://dx.doi.org/10.17118/11143/9921 
Page vide laissée intentionnellement. 


\section{AFFAIRE DE LA NOMINATION DU JUGE MAINVILLE À LA COUR D'APPEL DU QUÉBEC : UNE CONCESSION ET TROIS CRITIQUES}

par Maxime ST-HILAIRE*

Dans le jugement qu'elle a rendu oralement le 24 avril 2015 par la bouche du juge Wagner, la Cour suprême du Canada a rapidement rejeté le pourvoi formé contre l'avis de la Cour d'appel du Québec du 23 décembre 2014 selon lequel la nomination, à titre de membre de celle-ci, d'un candidat qui jusqu'alors était juge à la Cour d'appel fédérale, n'était pas inconstitutionnelle en vertu de l'article 98 de la Loi constitutionnelle de 1867. Au moyen de trois critiques de l'avis de la Cour d'appel du Québec, le texte qui suit vise à la démonstration du fait que cette affaire méritait, de la part de la Cour suprême, un véritable délibéré.

In a judgment delivered orally by Mr. Justice Wagner the 24th of April 2015, the Supreme Court of Canada quickly dismissed an appeal from a decision rendered the 23 $3^{\text {rd }}$ of December 2014 by the Quebec Court of Appeal, which decided that the nomination of a judge of the Federal Court of Appeal to the Supreme Court was not in violation of section 98 of the Constitution Act 1867. On the basis of three criticisms raised by the Court of Appeal, the article posits that this matter should have been taken under deliberation by the Supreme Court.

* $\quad$ Professeur adjoint, Faculté de droit, Université de Sherbrooke. 
Affaire de la nomination du juge Mainville

à la Cour d'appel du Québec : $\quad$ (2015) 45 R.D.U.S. une concession et trois critiques

"Nous sommes tous d'avis que le pourvoi doit être rejeté, essentiellement pour les motifs exposés par la Cour d'appel du Québec. [...] Les arguments fondés sur le Renvoi relatif à la Loi sur la Cour suprême [...] ne résistent pas à l'analyse. Comme la Cour d'appel l'a indiqué [...], le présent pourvoi concerne des dispositions constitutionnelles et législatives différentes et le raisonnement et les conclusions de ce renvoi ne s'appliquent pas en l'espèce ${ }^{1}$. "Voilà comment, par la bouche du juge Wagner, mais à l'unanimité, la Cour suprême a rapidement disposé de l'appel de l'avis de la Cour d'appel du Québec ${ }^{3}$ relatif à l'article 98 de la Loi constitutionnelle de $1867^{4}$ (ci-après "LC 1867 ").

Dans son avis du 21 mars 2014 sur sa loi constitutive ${ }^{5}$, la Cour suprême du Canada avait estimé que l'expression " choisis [...] parmi les avocats "de la province de Québec, à l'article 66, désignait les avocats actuels, à l'exclusion des anciens avocats ${ }^{7}$. Le 23 décembre de la même année, la Cour d'appel du Québec a avalisé la thèse selon laquelle l'article 98 LC 1867, où il est question de juges " selected from the Bar " (la traduction française non officielle parle de juges "choisis parmi les membres du barreau ") ${ }^{8}$, et l'article 6 de la Loi sur la Cour suprême, ne devaient pas recevoir la même interprétation.

La réponse à la question de la constitutionnalité, en vertu de l'article 98 LC 1867, de la nomination d'un juge de la Cour d'appel fédérale à la Cour d'appel du Québec pouvait dépendre de celle donnée à plusieurs sous-questions. La Cour d'appel du Québec n'a su me convaincre que sur l'une d'entre celles-ci, mais d'une manière insuffisante à me faire changer d'avis sur celle-là : une telle nomination devait être tenue pour inconstitutionnelle. Suivent donc

1. Québec (Procureure générale) c. Canada (Procureur général), 2015 SCC 22.

3. Renvoi sur l'article 98 de la Loi constitutionnelle de 1867 (Dans l'affaire du), 2014 QCCA 2365.

4. $\quad 30 \& 31$ Vict., c. 3 (R-U).

5. $\quad$ Renvoi relatif à la Loi sur la Cour suprême, art. 5 et 6, 2014 CSC 21.

6. Loi sur la Cour suprême, L.R.C. 1985, c. S-26.

7. Renvoi relatif à la Loi sur la Cour suprême, art. 5 et 6, préc., note 5, par. 37.

8. "Les juges des cours de Québec seront choisis parmi les membres du barreau de cette province." 
Affaire de la nomination du juge Mainville à la Cour d'appel du Québec :

une concession, puis trois critiques de l'avis de la Cour d'appel qu'a cautionné la Cour suprême et dans lequel je vois justement trois grands ordres de motifs : textuel, historique et pratique.

\section{Concession. Il est vrai que les "Courts of Quebec " au sens de l'article 98 LC 1867 ne sont que la Cour supérieure et la Cour d'appel}

Dans mon billet du 24 juin 20149, ainsi que dans la lettre ouverte qu'avec mon collègue le professeur Hugo Cyr je faisais paraître le lendemain dans Le Devoir ${ }^{10}$, j'affirmais que " ni le texte ni le contexte " de l'article 98 LC 1867 " ne restreint son champ d'application aux seuls juges des cours supérieures au sens large c'est-à-dire des cours supérieures (au sens strict) et d'appel ". Je soutenais ainsi qu'à l'article 98 LC 1867, " juges du Québec " voulait bel et bien dire "juges du Québec ", en ayant à l'esprit les membres de tous les tribunaux judiciaires de la province.

Cette interprétation n'est plus celle que je favoriserais. Elle voulait se justifier surtout par le compromis pratique auquel elle devait permettre d'en arriver en préservant la constitutionnalité de la nomination de membres de tribunaux judiciaires de compétence inférieure, dont la Cour du Québec, aux cours de justice de compétence supérieure. Or, puisque l'article " équivalent " - car il s'en distingue aussi par sa vocation transitoire d'origine - relatif aux autres provinces, l'article $97^{11}$, prévoit expressément ne s'appliquer

9. Maxime ST-HILAIRE, "Le festival harpérien de l'inconstitutionnalité se poursuit : la nomination du juge Mainville à la Cour d'appel du Québec ", Double Aspect, 24 juin 2014, en ligne : https:/ /doubleaspectblog.wordpress.com/2014/06/24/le-festivalharperien-de-linsconstitutionnalite-se-poursuit-la-nomination-du-jugemainville-a-la-cour-dappel-du-quebec/.

10. Maxime ST-HILAIRE, "Une menace pour la culture juridique québécoise ", Le Devoir, 25 juin 2014, en ligne : http://www.ledevoir.com/politique/ quebec/411814/nomination-du-juge-mainville-une-menace-pour-laculture-juridique-quebecoise.

11. "Jusqu'à ce que les lois relatives à la propriété et aux droits civils dans Ontario, la Nouvelle-Écosse et le Nouveau-Brunswick, et à la procédure dans les cours de ces provinces, soient rendues uniformes, les juges des cours de ces provinces qui seront nommés par le gouverneur-général 
Affaire de la nomination du juge Mainville

à la Cour d'appel du Québec : $\quad$ (2015) 45 R.D.U.S.

une concession et trois critiques

qu'aux juges nommés par le gouverneur général, je cède désormais devant l'objection de Léonid Sirota selon laquelle il serait problématique que "provincial court judges [be] ineligible for elevation to Superior Courts in common law provinces even if they are not in Québec "12. Et sur le plan historique, il est vrai que, à l'époque, de trop nombreux juges des juridictions québécoises de compétence inférieure, dont les juges de paix et les " commissaires ", étaient des non-juristes pour qu'il fût l'intention $\mathrm{du}$ constituant de disposer autrement. Je me range donc maintenant derrière l'opinion - qui était aussi celle, non seulement du Procureur général du Canada ${ }^{13}$, mais aussi de la Procureure générale du Québec ${ }^{14}$ - selon laquelle, tout comme l'article 97 pour les autres provinces, l'article 98 ne concerne, pour le Québec, que les nominations judiciaires prévues à l'article 96, soit celles des membres des cours " provinciales " de compétence supérieure, soit, à l'heure actuelle, la Cour supérieure et la Cour d'appel15.

Cette concession faite, il me restait à déterminer si l'on avait su me convaincre du fait que la meilleure interprétation à donner à

devront être choisis parmi les membres des barreaux respectifs de ces provinces."

12. Leonid Sirota, "The Mainville Appointment Is Constitutional ", Double Aspect, 26 juin 2014, en ligne : https://doubleaspectblog.wordpress. com/2014/06/26/the-mainville-appointment-is-constitutional/.

13. PROCUREUR GÉNÉRAL DU CANADA, "Renvoi relatif à l'article 98 de la Loi constitutionnelle de 1867 et aux conditions de nomination des juges des cours du Québec ", mémoire à la Cour d'appel du Québec, dossier no 500 09-024618-142, 2014, par. 40 et 53 (ci-après "mémoire PGC devant CAQ "); PROCUREUR GÉNÉRAl DU CANADA, "Procureure générale du Québec, et al. c. Procureur général du Canada ", mémoire à la Cour suprême du Canada, dossier no 36231, 2015, par. 28 et 36 (ci-après " mémoire PGC devant CSC ").

14. PROCUREUR GÉnÉRAL DU QuÉBEC, "Renvoi relatif à l'article 98 de la Loi constitutionnelle de 1867 et aux conditions de nomination des juges des cours du Québec ", mémoire à la Cour d'appel du Québec, dossier no 50009-024618-142, 2014, par. 46 (ci-après "mémoire PGQ devant CAQ "); PROCUREUR GÉNÉRAL DU QUÉBEC, "Procureure générale du Québec, et al. c. Procureur général du Canada ", mémoire à la Cour suprême du Canada, dossier no 36231, 2015, par. 61 (ci-après " mémoire PGQ devant CSC ").

15. Renvoi sur l'article 98 de la Loi constitutionnelle de 1867 (Dans l'affaire du), préc., note 3, par. 25. 
Affaire de la nomination du juge Mainville à la Cour d'appel du Québec :

l'expression " selected from the Bar " est celle qui lui fait dire " choisi par ceux qui ont déjà été admis au barreau aux fins de l'exercice de la profession d'avocat " plutôt que "choisi parmi les membres du barreau qui sont autorisés à exercer la profession d'avocat ".

\section{Première critique. "From the Bar " comme voulant dire " ayant déjà été admis au barreau ": réfutation de l'argument de texte}

L'argument de texte se résume comme suit. Dans la Loi sur la Cour suprême ${ }^{16}$, l'expression "parmi les avocats " de l'article 6 relatif à la nomination des juges provenant du Québec se distingue, surtout dans la version anglaise, de l'expression " is or has been [...] a barrister or advocate "de l'article 5 relatif à la nomination des juges provenant des autres provinces. Partant, à défaut de pouvoir se distinguer d'une expression équivalente à "is or has been " à l'article 97 LC 1867 relatif à la nomination des juges des cours supérieures des autres provinces, l'expression " selected from the Bar " de l'article 98 LC 1867 relatif à la nomination des juges des cours supérieures du Québec veut forcément dire "choisis parmi les membres actuels ou anciens ". Bref, la Cour d'appel est d'avis que, en principe, une expression telle que "parmi les avocats de..." ou " parmi les membres du barreau de..." veut aussi renvoyer aux anciens avocats ou membres du barreau ${ }^{17}$. Ce ne serait donc qu'exceptionnellement qu'une telle expression pourrait ne vouloir dire que ce qu'elle dit. En plus d'être hautement contre-intuitif, cet " argument " n'en est pas un. Il est arbitraire. Il érige le particulier et contingent en général et nécessaire. Qui plus est, il est anachronique, car cette logique textuelle de la Loi sur la Cour suprême18 ne remonte qu'à 1886 . Je m'explique.

En 1886, la loi sur la Cour suprême introduit, pour les candidats à la charge de juge de cette cour, une distinction entre la catégorie de seuls avocats actuels (à l'exclusion des anciens

16. Préc., note 6.

17. Renvoi sur l'article 98 de la Loi constitutionnelle de 1867 (Dans l'affaire du), préc., note 3, par. 69.

18. Préc., note 6. 
Affaire de la nomination du juge Mainville

à la Cour d'appel du Québec :

(2015) 45 R.D.U.S.

une concession et trois critiques

avocats) et celle d'avocats anciens ou actuels ${ }^{19}$. Convaincue facilement par le Procureur général du Canada, la Cour d'appel fût d'avis que, par contraste, une telle distinction était pertinente pour comprendre le sens de l'expression "from the Bar "à l'article 98 LC $1867^{20}$. Il s'agit ici d'une pétition de principe. Cette pertinence n'avait rien d'évident, mais devait être démontrée. La loi (à l'époque ordinaire) de 1886 et relative à d'autres nominations judiciaires vient faire une distinction que ne fait pas la Loi constitutionnelle de 1867. En quoi cela devait-il rétroagir sur le sens à donner à la loi constitutionnelle? Personne ne l'a expliqué. Plus encore, même si elle était pertinente, la comparaison entre l'absence d'une distinction dans la Loi constitutionnelle de 1867 et sa présence dans une loi ordinaire de 1886 pourrait très bien être interprétée en un sens différent. La Cour d'appel " décrète ", pour ainsi dire, que si on distingue entre la catégorie de seuls avocats actuels et celle d'avocats anciens ou actuels en 1886, cela veut forcément dire que, 19 ans auparavant, concernant d'autres nominations judiciaires dans une loi constitutionnelle, l'expression "from the Bar " voulait forcément correspondre à une catégorie équivalente à cette dernière. Comme si ce qui était nouveau en 1886 concernant les nominations à la Cour suprême était évidemment la catégorie des avocats actuels, et ne pouvait pas être (concernant les nominations des juges autres que québécois) celle des anciens avocats. Il est même un argument allant en sens inverse pour suggérer que, en 1886, c'était plutôt cette dernière catégorie qui était nouvelle. Il s'agit de l'avis de la CSC dans l'affaire du juge Nadon, où la majorité a conclu que, en vertu de la loi antérieure sur la CSC, celle de 1875, " seules les personnes étant avocats au moment de leur nomination pouvaient être nommées à la Cour, tant pour le Québec que pour le reste du pays ${ }^{21}$. Deuxième pétition de principe, donc.

19. Acte des cours Suprême et de l'Échiquier, S.R.C. 1886, c. 135, art. 4(2) et (3).

20. Renvoi sur l'article 98 de la Loi constitutionnelle de 1867 (Dans l'affaire du), préc., note 3, par. 29.

21. Renvoi relatif à la Loi sur la Cour suprême, art. 5 et 6, préc., note 5, par. 21 . 
(2015) 45 R.D.U.S.

Affaire de la nomination du juge Mainville

à la Cour d'appel du Québec :

581

une concession et trois critiques

\section{Deuxième critique. "From the Bar" comme voulant dire " ayant déjà été admis au barreau " : réfutation de l'argument historique}

Quant à l'argument d'ordre historique, il veut que des objectifs de nature différente président à l'article 6 de la Loi sur la Cour suprême 22 et à l'article 98 LC 1867. De l'avis de la Cour d'appel du Québec, lors des débats sur le projet de fédération, les "représentants du Bas-Canada auraient pu craindre qu'en accordant un pouvoir de nomination aussi important au gouverneur général, la pratique de nommer des juges d'origine étrangère, ou dénués de connaissance en droit privé local, reprendrait de plus belle. Mais, à une exception près, ce ne fut pas le cas "23. S’il fallait en croire la Cour d'appel, "l'article 98 [de la LC 1867] s'inscri[rait] dans un contexte précis et bien différent de celui qui entoura la création de la Cour suprême " ${ }^{24}$. Rien n'est plus faux. En effet, dès lors qu'il fut proposé que le gouverneur général soit chargé de la nomination des juges des " cours supérieures " des provinces, les élites du Bas-Canada se sont inquiétées. C'est en réponse aux questions des députés que, le 21 février 1865, le solliciteur général du Canada-Uni, Sir Hector-Louis Langevin, intervient en assemblée pour dire que, "dans la constitution proposée, il y a un article qui porte que les juges des cours du BasCanada seront choisis parmi les membres du barreau de cette section [...]"25. Il se trompe toutefois en avançant que "[c]ette exception n'a été faite que pour le Bas-Canada "26. Avant la résolution no 35 des 72 Résolutions de Québec d'octobre 1864, qui prévoyait que "[t]he judges of the courts of Lower Canada shall be selected from the bar of Lower Canada ", se trouvait la résolution no 34, qui prévoyait que, "[u]ntill the consolidation of the laws of Upper Canada, New Brunswick, Nova Scotia, Newfoundland, and

22. Préc., note 6.

23. Renvoi sur l'article 98 de la Loi constitutionnelle de 1867 (Dans l'affaire du), préc., note 3 , par. 50.

24. Id., par. 52.

25. Canada, Assemblee legislative, Débats parlementaires sur la question de la Confédération des provinces de l'Amérique britannique du Nord, 3e sess., 8e Parl., 21 février 1865, p. 394.

26.

Id. 
Affaire de la nomination du juge Mainville
à la Cour d'appel du Québec :
(2015) 45 R.D.U.S.

une concession et trois critiques

Prince Edward Island, the judges of these provinces appointed by the general government [sic] shall be selected from their respective bars „27. On y reconnait aisément les futurs articles 97 et $98 \mathrm{LC}$ 1867. Or, la disposition concernant les provinces autres que le Québec fait dans les deux cas allusion à l'uniformisation prévue du droit des autres provinces (résolution no 29.3328, futur article 94 LC 1867) et se présente donc comme disposition de droit transitoire. C'est à bon droit que la Procureure générale du Québec a voulu rappeler à la Cour suprême que, "[d]e fait, dès la Conférence de Québec, certains Pères de la Confédération ont manifesté le souhait d'avoir un système de droit uniforme et un seul barreau pour tout le Canada. La réalisation d'un tel objectif était, toutefois, impossible étant donné la position du Bas-Canada "29. Si l'adoption de l'article 96 LC 1867 a généré peu de débats, c'est que l'article 98 venait en limiter les risques, non pas que la question avait perdu de son importance. Compris notamment à la lumière des conditions de nomination à la Cour supérieure et à la Cour du Banc de la Reine (ancêtre de la Cour d'appel du Québec comme cour permanente) qui étaient prévues dans les lois du Canada-Uni qui devaient être transitoirement reconduites, cet article 98 était tenu pour clair. De distinguer les enjeux de l'article 98 de la LC 1867 de ceux des dispositions qui, dans l'histoire de la Cour suprême, ont assuré à celle-ci un contingent de juges québécois, relève de la fabrication.

La lutte pour la " représentation du droit québécois " au sein de la composition de la Cour suprême du Canada s'est inscrite dans

27. Report of resolutions adopted at a conference of delegates from the provinces of Canada, Nova Scotia, and New Brunswick, and the colonies of Newfoundland and Prince Edward Island, held at the city of Quebec, October 10, 1864, as the basis of a proposed confederation of those provinces and colonies, reprod. in Janet AJZENSTAT, Paul RoMNEY, Ian Gentles et William D. GAIRDNER (dir.), Canada's Founding Debates, Toronto, University of Toronto Press, 1999, "Appendix A ", p. 465-472, à la page 468 .

28. Id.

29. Mémoire PGQ devant CSC, préc., note 14, par. 50. 
le prolongement direct de celle qui, de longue haleine ${ }^{30}$, avait mené aux acquis que visait à préserver l'article 98 LC 1867. Dès 1865, la possibilité projetée (par la résolution no 29.3431) que, au sein de la fédération des colonies, le nouveau parlement fédéral puisse créer une " cour générale d'appel " inquiète par exemple le député HenriElzéar Taschereau : "Nous avons la garantie que nous aurons nos tribunaux locaux, que nos juges seront pris parmi les membres du barreau du Bas-Canada, et que nos lois civiles seront maintenues; mais pourquoi établir une cour d'appel fédérale dans laquelle il y aura appel des décisions rendues par tous nos juges [...]? „32. Après l'entrée en vigueur de la LC 1867, la création d'une telle cour sera d'abord proposée en $1869^{33}$, puis en $1870^{34}$, avant de devenir réalité en 1875, par une loi constitutive prévoyant que deux de ses six juges " seront pris parmi les juges de la Cour supérieure ou de la Cour du Banc de la Reine, ou parmi les avocats de la province de Québec "35. Il est évident que, en l'étendant à ce nouvel échelon de la hiérarchie judiciaire, c'est la logique de l'article 98 LC 1867 qui est reprise ici. Il ne fait selon moi aucun doute que la Procureure générale du Québec était dans le vrai en affirmant qu'il " ressort des débats entourant la création de la Cour suprême que c'est la crainte que la garantie dont bénéficiait [sic] les cours supérieures du Québec en vertu de l'article 98 LC 1867 soit contrecarrée par l'établissement d'un nouveau tribunal d'appel qui a incité les représentants du Québec à exiger une représentation de juges du Québec au sein de la Cour suprême. [...] Avec égards, la Cour d'appel insiste à tort sur le fait que l'article 6 de la Loi sur la Cour suprême $e^{36}$ consacre un compromis historique qui serait limité à la création de la Cour suprême "37.

30. Evelyn Kolish, Nationalismes et conflits de droits : le débat du droit privé au Québec 1760-1840, Montréal, Hurtubise HMH, 1994.

31. Préc., note 31.

32. Mémoire PGQ devant CSC, préc., note 14, par. 104.

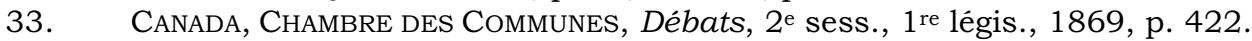

34. Canada, Chambre des Communes, Débats, 3e sess., 1re légis., 1870, p. 502.

35. Acte de la Cour suprême de l'Échiquier pour le Canada, S.C. 1875, c. 11, art. 4.

36. $\quad$ Préc., note 6.

37. Mémoire PGQ devant CSC, préc., note 14, par. 111-112; voir aussi Mémoire PGQ devant CAQ, préc. note 14, par. 113. 
Affaire de la nomination du juge Mainville
à la Cour d'appel du Québec :
(2015) 45 R.D.U.S.

une concession et trois critiques

Or la prise en compte de l'histoire fondait un argument bien plus fort, que la Cour d'appel n'a fait qu'évoquer ${ }^{38}$, mais que le Procureur général du Canada avait brillamment exposé 39 . Cet argument est que la catégorie de "membre du barreau " d'une province est définie, non pas par la loi constitutionnelle, mais par la loi ordinaire provinciale et les règlements des différents barreaux provinciaux, de sorte que, dans l'histoire - et encore aujourd'hui d'une province à l'autre - les juges, ou certains d'entre eux, ont pu conserver, tel quel ou modifié, leur statut de membre du barreau après leur nomination. Partant, en 1867, l'expression " selected from the Bar " aurait été appelée à couvrir les juges des cours de compétence inférieure qui continuaient d'appartenir au barreau de la province. Relevons en passant que ce n'est que depuis 1967, et ce, par inadvertance apparemment, que, avec l'abolition du statut de membre honoraire, la loi québécoise relative au barreau prive les juges de toute appartenance à cet ordre professionnel ${ }^{40}$. Dans leur mémoire devant la Cour d'appel, les procureurs des Cris soutenaient ainsi " qu'au moment de la rédaction de l'article 98 de la Loi constitutionnelle de 1867, les juges étaient considérés comme étant toujours des membres du Barreau du Bas-Canada " 41 . Faisant encore un pas de plus, ils, ainsi que le Procureur général du Canada, ont réussi à convaincre la Cour d'appel du Québec de ce que, à l'article 98 LC 1867, "from the Bar " voulait dire " ayant déjà été admis à l'exercice de la profession d'avocat par le barreau ". L'argument a presque réussi à me convaincre.

Or, en 1867, les mots " selected from the Bar ", à l'article 98 LC 1867, devaient manifestement être compris à la lumière des conditions de nomination à la Cour supérieure et à la Cour du Banc

38. Renvoi sur l'article 98 de la Loi constitutionnelle de 1867 (Dans l'affaire du), préc., note 3, par. 61.

39. Mémoire PGC devant CAQ, préc., note 13, par. 65 à 84; voir aussi Mémoire PGC devant CSC, préc., note 13, par. 51-53.

40. Mémoire PGC devant CAQ, préc., note 13, par. 82-84; voir aussi Mémoire PGC devant CSC, préc., note 13, par. 51-52.

41. GRAND CONSEIL DES CRIS ET GOUVERNEMENT DE LA NATION CRIE, " Renvoi relatif à l'article 98 de la Loi constitutionnelle de 1867 et aux conditions de nomination des juges des cours du Québec ", mémoire à la Cour d'appel du Québec, dossier no 500-09-024618-142, 2014, par. 63. 
de la Reine du Bas-Canada, conditions qui étaient prévues dans les lois du Canada-Uni, dont l'article 129 LC 1867 prévoyait, de façon transitoire, le maintien en vigueur. Et il se trouve que ces conditions opposaient, comme catégories de candidats, celle des membres du barreau à celle de juges de tribunaux précisément indiqués. Cela exclut donc une catégorie de juges d'autres juridictions ayant conservé une forme ou une autre d'appartenance au barreau qui, au Bas-Canada, n'a été constitué par la loi qu'en 184942. En effet, l'article 4 de l'Acte pour amender les lois relatives aux cours de juridiction civile en première instance dans le Bas-Canada ${ }^{43}$, qui porte création de la Cour supérieure - nom qui provient sans doute, comme le suggère Donald Fyson ${ }^{44}$, des anciens " superior terms " de la Cour du banc du roi (ou de la reine) - prévoyait " qu'aucune personne ne sera nommée juge de la dite cour supérieure, à moins qu'immédiatement avant nomination elle ne soit juge de l'une des dites cours du banc de la reine, ou juge de circuit ou de district, ou avocat de dix ans de pratique au moins au barreau du BasCanada ". La mention de juge de district m'intrigue, car à ma connaissance, avec les Cours de Division, les Cours de District avaient été abolies en $1843^{45}$. Quant à l'Acte pour établir une cour ayant juridiction en appel et en matières criminelles pour le BasCanada ${ }^{46}$, qui portait création de la Cour du Banc de la Reine du Bas-Canada en tant que cour d'appel permanente, son article 2 prévoyait que "personne ne sera nommé juge-en-chef ou juge puisné comme susdit, à moins d'avoir été, lors de sa nomination,

42. Acte pour l'incorporation du Barreau du Bas-Canada, S.C. 1849, 12 Vict., c. 46.

43. $\quad$ S.C. 1849,12 Vict., c. 38.

44. David Fyson, "Court Profiles : Superior Court ", The Court Structure of Quebec and Lower Canada, 1764 - 1860, Montreal History Group, 2012, en ligne : http://www.profs.hst.ulaval.ca/Dfyson/Courtstr/superior.htm

45. Acte pour abroger certains Actes et Ordonnances y mentionnés et pour mieux pourvoir à l'administration de la Justice dans le Bas-Canada, S.C. 1843, 7 Vict., c. 16; Acte pour établir une meilleure Cour d'appel dans le Bas-Canada, S.C. 1843, 7 Vict., c. 18; Acte pour pourvoir à la décision sommaire des Petites Causes dans le Bas-Canada, S.C. 1843, 7 Vict., c. 19. Voir Pierre-Georges Roy, Les juges de la Province de Québec, Québec, Service des Archives du gouvernement de la Province de Québec, 1933, p. xvi.

46. S.C. 1849 , c. 37. 
Affaire de la nomination du juge Mainville

à la Cour d'appel du Québec : $\quad$ (2015) 45 R.D.U.S. une concession et trois critiques

juge de l'une des diverses cours du banc de la Reine dans le BasCanada, ou juge de la cour supérieure, ou juge de circuit, ou à moins d'avoir été avocat pratiquant pendant au moins dix ans au barreau du Bas-Canada [...]". Ici, malgré une rédaction moins habile, "avoir été avocat " devait aussi se vérifier au moment de la nomination, et "pendant " voulait dire "depuis". La version anglaise parle d'ailleurs d'un "Advocate of at least ten years' standing at the Bar of Lower-Canada".

Au-dessous de la Cour supérieure venait en effet la Cour de circuit, alors héritière de la compétence de l'ancienne Cour du Banc de la Reine "en termes inférieurs ". En 1857, l'article 13 de l'Acte pour amender les actes de judicature du Bas Canada ${ }^{47}$ abolit la charge de juge de circuit et prévoit que la Cour de circuit sera tenue par les juges de la Cour supérieure qui "auront tous les pouvoirs et les devoirs accordés et attribués à tout juge de circuit à l'époque à laquelle la présente section sera mise à effet ". Cela ne suffit pas pour dire que la Cour de circuit, dont la compétence est large mais d'attribution, devient pour autant une cour de compétence supérieure. Or, la Cour de circuit disposera d'un pouvoir de surveillance et de contrôle, pouvoir que la jurisprudence canadienne postérieure à la fédération de 1867 considèrera être propre aux cours de compétence supérieure. La doctrine et la jurisprudence ${ }^{48}$ canadiennes semblent unanimes à voir une telle cour de compétence supérieure dans la nouvelle forme que prend la Cour de circuit, qui sera abolie par le législateur provincial en 1952, alors que sa compétence sera attribuée à la Cour (provinciale inférieure) de magistrat, et ce, en vertu d'une loi de 194549. De 1867 à son abolition en 1952, la Cour de circuit aurait donc été une " cour de district " au sens de l'article 96 LC 186750. Mais si ce fût le cas, alors il demeure difficile de s'expliquer comment cette juridiction a pu être validement abolie par la loi ordinaire

47. S.C. 1857,20 Vict., c. 44.

48. $\quad$ Séminaire de Chicoutimi c. La Cité de Chicoutimi, [1973] R.C.S. 681, p. 689692. Voir aussi : Renvoi touchant la constitutionnalité de la Loi concernant la juridiction de la Cour de magistrat, [1965] R.C.S. 772.

49. S.Q. 1945, 9 Geo VI, c. 19.

50. Séminaire de Chicoutimi c. La Cité de Chicoutimi, préc., note 48, p. 691. 
Affaire de la nomination du juge Mainville

à la Cour d'appel du Québec :

provinciale. D'ailleurs, des lois québécoises de 1888 et 1889 , portant respectivement abolition de la Cour de circuit dans le district de Montréal et attribution exclusive, dans ce même district, de sa compétence à la Cour de magistrat, ont été successivement désavouées par le gouverneur général ${ }^{51}$. Quoi qu'il en soit du statut de cette nouvelle Cour de circuit dans la hiérarchie judiciaire, et nonobstant le fait qu'elle abolit la charge de juge de circuit, la loi de 1857 prévoit de manière transitoire, à son article 10, que "les nouveaux juges de la cour [supérieure] et tous les juges qui y seront nommés à l'avenir seront choisis parmi les juges de circuit d'alors et les avocats de dix années de pratique au moins dans le barreau du Bas-Canada [...] ". La catégorie des juges de district disparaît, et celle des juges de circuit est appelée à le faire.

À l'occasion de la refonte de 1861, l'Acte concernant la Cour supérieure ${ }^{52}$ est mis à jour, et son nouvel article 7 , relatif aux conditions de nomination, se lit comme suit : "Le juge en chef et les juges de la cour supérieure, en office lors de la mise à effet de la section neuf de l'acte 20 V. c. 44, continuent à l'être en vertu de la commission qu'ils avaient alors; les nouveaux juges de la cour ont été choisis parmi les juges de circuit d'alors, et les avocats de dix années de pratique au moins au barreau du Bas Canada;- et tous les juges qui seront nommés à l'avenir seront choisis parmi les dits avocats ayant pratiqué pendant le même nombre d'années." Désormais, seuls les avocats en exercice depuis dix ans au Barreau du Bas-Canada seront éligibles à la charge de juge de la Cour supérieure du Bas-Canada. Quant à l'Acte concernant la Cour du Banc de la Reine 53 , son nouvel article 2 se lit comme suit : "Nul ne sera nommé juge-en-chef ou juge puisné, à moins d'avoir été, lors de sa nomination, juge de la Cour supérieure du Bas Canada, ou à moins d'avoir été avocat pratiquant pendant au moins dix ans au

51. Acte amendant la loi relative aux magistrats de district, S.Q. 1888, 51-52 Vict., c. 20; Acte amendant la loi relative aux magistrats de district, S.Q. 1889, 52 Vict., c. 30. Voir Jacques DESLAURIERS, "La Cour provinciale et l'art. 96 de 1'A.A.N.B ", (1997) 18 C. de D. 881, p. 888-891. Voir aussi Sylvio NoRmAnd, La Cour du Québec : genèse et développement, Montréal, Wilson \& Lafleur, 2013, p. 4.

52. S.R.B.-C. 1861, c. 78 .

53. S.R.B.-C. 1861 , c. 77. 
Affaire de la nomination du juge Mainville

à la Cour d'appel du Québec :

(2015) 45 R.D.U.S. une concession et trois critiques

barreau du Bas Canada. " La formulation est la même qu'en 1849, à cette exception près que les catégories de candidats formées des juges des anciennes et diverses cours du banc de la Reine du BasCanada et des juges de circuit sont abolies. En 1864, une cour d'appel intermédiaire, devant prendre place entre la Cour supérieure et la Cour du Banc de la Reine, est créée : la Cour de révision, qui sera abolie en $1920^{54}$. L'article 20 de sa loi constitutive prévoit cependant qu'elle se composera de "trois juges de la cour supérieure à Montréal ou à Québec " 55 , de sorte que cette réorganisation judiciaire est sans conséquences sur les conditions de nomination aux tribunaux auxquels s'appliquera bientôt l'article 98 LC 1867. Nous y voilà : la thèse selon laquelle l'expression " from the Bar ", à cet article, serait alors comprise comme voulant dire " détenant une forme ou une autre de statut de membre du barreau (dont celui que pourrait avoir le membre d'un tribunal non désigné par les lois du Canada-Uni relatives aux conditions de nomination à la Cour supérieure et à la Cour du Banc de la Reine du BasCanada)" se révèle des plus improbables. Cette thèse ne peut aucunement militer en faveur de celle selon laquelle, de nos jours, l'expression qui nous occupe devrait vouloir dire "ayant déjà été admis à l'exercice de la profession d'avocat par le barreau ".

La Procureure générale du Québec était du reste fondée de souligner que, dans les premières décennies qui ont suivi l'entrée en vigueur de la LC 1867, le Parlement fédéral n'a pas jugé utile de modifier les conditions de nomination des juges de la Cour supérieure et de la Cour du Banc de la Reine du Québec qui étaient posées par les lois du Canada-Uni56. Avec les autres dispositions des lois du Canada-Uni dont le contenu relevait maintenant de la compétence du Parlement fédéral, celles qui nous intéressent ici n'ont été abrogées qu'à la faveur de la refonte de $1886^{57}$. Qui plus est, elles n'ont été remplacées par le législateur fédéral qu'en

54. Loi relative à l'organisation et à la compétence des tribunaux de juridiction civile et à la procédure, en certains cas, S.Q. 1920, 10 Geo. V, c. 79.

55. S.C. $1864,27-28$ Vict., c. 39.

56. Mémoire PGQ devant CSC, préc., note 14, par. 77.

57. Acte concernant les statuts révisés du Canada, S.C. 1886, 49 Vict., c. 4. 
Affaire de la nomination du juge Mainville

à la Cour d'appel du Québec :

191258. Qu'on me comprenne bien : je ne suis pas en train de dire que ces conditions " préconfédératives " de nomination aux cours de compétence supérieure du Québec ont été constitutionnalisées dans le détail par l'article 98 LC 1867. Je suis plutôt en train de dire que c'est à leur lumière que s'éclaire d'abord le sens de l'expression "from the Bar " qu'on trouve à ce dernier article. Et les paragraphes qui précèdent ne laissent guère douter du fait que c'est la Procureure générale du Québec qui avait raison en soutenant que, " au moment de la Confédération, les conditions de nomination aux tribunaux supérieurs du Québec étaient bien établies. Seuls les membres du Barreau du Bas-Canada et des tribunaux supérieurs de la province pouvaient y accéder "59. La Cour d'appel du Québec reconnaît elle-même dans son avis que, "pendant la période préconfédérative, seuls les juristes qui depuis dix ans étaient avocats en exercice, ou qui étaient juges de la Cour supérieure, de district ou de comté, pouvaient devenir juges à la Cour du Banc de la Reine "60.

Relevons au passage que, si, de la part de la Cour d'appel, d'interpréter l'article 98 LC 1867 à la lumière des lois du CanadaUni qui en constituaient le contexte historique s'imposait et n'aurait pas été une erreur, en revanche, d'interpréter ce même article constitutionnel à la lumière des dispositions de la loi ordinaire postérieure à l'entrée en vigueur de la LC 1867, dispositions dont cet article qui nous occupe fonde les conditions de validité, en était $u^{6} e^{61}$. Je partage donc aisément l'opinion de la Procureure générale du Québec selon laquelle, " contrairement à ce qu'avance l'avis de la Cour d'appel, une loi fédérale ne peut servir à interpréter une disposition constitutionnelle dont le rôle est d'encadrer le pouvoir du gouvernement fédéral sur une institution provinciale "62. Il s'agit ici d'une notion élémentaire et universelle de droit constitutionnel.

58. Loi modifiant la Loi des juges, S.C. 1912, 2 Geo. V, c. 29, art. 9.

59. Mémoire PGQ devant CSC, préc., note 14, par. 71; voir aussi Mémoire PGQ devant CAQ, préc., note 14, par. 90.

60. Renvoi sur l'article 98 de la Loi constitutionnelle de 1867 (Dans l'affaire du), préc., note 3, par. 64 .

61. Id., par. 58-67.

62. Mémoire PGQ devant CSC, préc., note 14, par. 97. 
Affaire de la nomination du juge Mainville

à la Cour d'appel du Québec : $\quad$ (2015) 45 R.D.U.S. une concession et trois critiques

Encore là, contrairement à la reconstruction à laquelle la Cour d'appel se livre à l'invitation du Procureur général du Canada63, l'histoire de la loi fédérale relative aux conditions de nomination aux cours de compétence supérieure n'est pas de continuité, mais de rupture. Ce n'est que depuis 1976 que, outre les avocats de la province, d'anciens avocats de la province devenus juges de la cour provinciale ou de la Cour suprême du Yukon ou des Territoires du Nord-Ouest peuvent être nommés aux cours de compétence supérieure de cette même province ${ }^{64}$. Quant aux avocats de la province qui sont devenus juges des cours fédérales, ils ne le peuvent que depuis une modification apportée à la loi en 1996, de manière à inclure les anciens avocats de la province " ayant exercé à temps plein des fonctions de nature judiciaire à l'égard d'un poste occupé en vertu d'une loi fédérale ou provinciale "65.

\section{Troisième et dernière critique. "From the Bar " comme voulant dire " ayant déjà été admis au barreau " : réfutation (partielle) de l'argument pratique}

La Cour d'appel du Québec et, à sa suite, la Cour suprême du Canada, faisaient face à une réalité pratique qu'elles ont pu tenir pour contraignante : "Ce ne sont pas tous les juges de toutes les cours supérieures canadiennes, en première instance ou en appel, qui ont accédé à la magistrature en provenance directe du barreau d'une province, loin de là. [...] Beaucoup ont été nommés à une cour supérieure après avoir exercé une fonction judiciaire dans un tribunal visé par le paragraphe 14 de l'article 92 de la Loi constitutionnelle de 1867 "66. Dans son billet du 26 juin 201467, Léonid Sirota donnait l'exemple de la juge Abella, qui, en 1992, avait été nommée à la Cour d'appel de 1'Ontario alors qu'elle était juge de

63. Mémoire PGC devant CAQ, préc., note 13, par. 88-99; voir aussi Mémoire PGC devant CSC, préc., note 13, par. 95-96 et 99-100.

64. Loi modifiant la Loi sur les juges et d'autres dispositions concernant la magistrature, S.C. 1976-1977, c. 25, art. 1.

65. Loi modifiant la Loi sur la Cour fédérale, la Loi sur les juges et la Loi sur la Cour canadienne de l'impôt, L.C. 1996, c. 22, art. 2.

66. Renvoi sur l'article 98 de la Loi constitutionnelle de 1867 (Dans l'affaire du), préc., note 3, par. 59.

67. Préc., note 12. 
la Cour provinciale de cette même province. Concernant le Québec, la Procureure générale de cette province a dû concéder que, "depuis 1867 , les juges des cours supérieures sont régulièrement choisis [non seulement] parmi les membres du Barreau, mais également parmi les membres des tribunaux judiciaires du Québec "68. Bref, il était devenu pratiquement impossible de reconnaître l'inconstitutionnalité d'un aussi grand nombre de nominations judiciaires, sur une période du reste aussi longue.

Toutefois, il en va autrement de la nomination, à une cour visée par l'article 97 ou par l'article 98 LC 1867, d'un juge fédéral ou territorial. Dans son billet du 14 juillet 201469, Paul Daly donnait l'exemple du juge Robertson qui, en 2000, avait été nommé juge de la Cour d'appel du Nouveau-Brunswick alors qu'il était juge de la Cour d'appel de la cour martiale du Canada. Or, le fait que la nomination d'un juge fédéral à une cour visée par les articles 97 ou 98 LC 1867 fût inusité devait normalement tomber à pic, compte tenu de l'avis de la Cour suprême du 21 mars 2014 relatif aux articles 5 et 6 de sa loi constitutive. Regrettablement, la Cour suprême ne l'a pas entendu de cette oreille.

Le droit n'est pas censé permettre que soit fait indirectement ce qu'il interdit de faire directement. La Cour suprême du Canada vient pourtant de permettre, en le faisant transiter par la Cour supérieure ou par la Cour d'appel du Québec, au gouverneur en conseil de nommer à cette même Cour suprême du Canada, à titre de juge du Québec, un (ancien) juge fédéral, ce dont elle nous avait dit que, en vertu de l'article 6 de la Loi sur la Cour suprême ${ }^{70}$ (constitutionnalisé par l'al. 41d) LC 1982), c'était impossible de faire

68. Mémoire PGQ devant CSC, préc., note 14, par. 81; voir aussi Mémoire PGQ devant CAQ, préc., note 14, par. 85-86.

69. Paul DALY, "Judicial Musical Chairs II : the Constitutionality of Robert Mainville's Appointment to the Quebec Court of Appeal ", Administrative Law Matters, 14 juillet 2014, en ligne : http://www.administrativelawmatters.com/blog/2014/07/14/judicial-muscialchairs-ii-the-constitutionality-of-robert-mainvilles-appointment-to-the-quebeccourt-of-appeal/.

70. Préc., note 6. 
Affaire de la nomination du juge Mainville

à la Cour d'appel du Québec : $\quad$ (2015) 45 R.D.U.S.

une concession et trois critiques

directement. Contrairement à la formule sommaire des motifs oraux du juge Wagner, il ne devait pas faire de doute, non seulement que " [1]es articles 98 LC 1867 et 6 de la Loi sur la Cour suprême ${ }^{71}$ sont deux dispositions constitutionnelles participant à la protection du système civiliste dans le cadre des nominations judiciaires par le gouverneur général " (seul ou en conseil), de sorte qu'il importait " de concilier leur interprétation de manière à assurer la cohérence de la structure constitutionnelle canadienne "72, mais aussi, et plus particulièrement, qu'il était capital de s'assurer que l'interprétation jurisprudentielle de l'article 98 LC 1867 ne permette pas de faire indirectement ce que celle de l'article 6 de la Loi sur la Cour suprême 73 ne permet pas de faire directement. Or, l'interprétation de l'article 98 LC 1867 qu'a d'avalisée la Cour suprême du Canada " pourrait miner significativement la confiance du public envers le système judiciaire canadien "74. On est certes autorisé à penser que la Cour suprême vient de désavouer son avis dans " l'affaire du juge Nadon ". On l'est encore davantage de craindre que c'est ce que pensera le grand public. Léonid Sirota aimerait objecter que :

the majority in l'Affaire Nadon did not say that a former judge of the federal courts can never be appointed to the Supreme Court. On the contrary, the majority specifically pointed out that it did "not address" the question of whether such judge "who was a former advocate of at least 10 years standing at the Quebec bar could rejoin the Quebec bar for a day in order to be eligible for appointment to this Court under s. 6" [71] - much less that of a judge served on one of Québec's courts for some substantial period of time ${ }^{75}$.

Voilà qui consiste à dire que, puisque la CSC ne s'est pas prononcée sur la constitutionnalité d'un moyen indirect de nommer à la CSC, comme juge du Québec, un juge d'une cour fédérale, il

71. Id.

72. Mémoire PGQ devant CSC, préc., note 14, par. 113; voir aussi Mémoire PGQ devant CAQ, préc., note 14, par. 115 et 117 .

73. Préc., note 6.

74. Mémoire PGQ devant CSC, préc., note 14, par. 125.

75. Leonid Sirota, "No, no, no!", Double Aspect, 3 mai 2015, en ligne: https://doubleaspectblog.wordpress.com/2015/05/03/no-no-no/. 
Affaire de la nomination du juge Mainville à la Cour d'appel du Québec :

fallait en déduire, soit qu'elle était délibérément disposée à cautionner procédé du genre (comme elle vient de le faire avec son avis dans l'affaire du juge Mainville), soit que, n'ayant pas explicitement interdit un tel procédé indirect plutôt que de ne pas se prononcer sur la question, il devenait impossible à un juriste de mobiliser un principe général de droit selon lequel on n'est pas censé pouvoir faire indirectement ce qu'il est interdit de faire directement. Ainsi mise au jour, cette objection se révèle arbitraire, sophistique, bref, anémique.

La solution proposée par la Procureure générale du Québec devant la Cour suprême était d'interpréter "from the Bar of that province ", à l'article 98 LC 1867, comme voulant dire "parmi les membres actuels du Barreau du Québec ou ceux des tribunaux judiciaires québécois "76. Une telle solution aurait d'ailleurs pu et dû être appliquée (mutatis mutandis, évidemment) à l'interprétation de l'article 97 LC 1867. Une telle solution aurait représenté un bien meilleur compromis entre le droit et les faits.

Concernant le soi-disant "curious result " de cette interprétation, soit que "while eligible under s. 5 of the Supreme Court Act to represent the province from which they were originally appointed to the federal courts at the Supreme Court (something the Supreme Court unanimously confirmed in l'Affare Nadon), federal court judges could not be appointed to that province's own courts under s. 97 "77, ma réponse est que, contrairement aux trois juges qui y "représentent " le Québec, les autres juges de la CSC n'y représentent pas une province donnée. Souvenons-nous d'ailleurs du projet consigné à l'article 94 LC 1867, et de la nature transitoire qui, partant, devait être celle de l'article 97 LC 1867. Mais même si l'on devait ne pas être d'accord avec moi sur ce point, j'aurais encore, je crois, quelque chose à répondre de manière subsidiaire. $\mathrm{Si}$ rien n'interdisait au législateur fédéral, avant la constitutionnalisation, en 1982, de la composition de la CSC, de rendre éligibles à titre de juge de cette haute cour, pour les juges

76. Mémoire PGQ devant CSC, préc., note 14, par. 97; voir aussi Mémoire PGQ devant CAQ, préc., note 14, par. 82.

77. Leonid SIROTA, préc., note 75. 
des provinces autres que le Québec, les anciens avocats (ce qui devait comprendre les juges fédéraux), rien ne l'obligeait à le faire. Il aurait bien pu, dans un esprit davantage compatible avec l'article 97 LC 1867, s'en tenir aux avocats actuels et juges des cours de compétence supérieure de la province " représentée " par le juge en question. C'est ainsi que, entre la loi ordinaire inconstitutionnelle et la loi ordinaire qui met le mieux en œuvre l'esprit de la constitution, se trouve parfois la loi ordinaire constitutionnelle, mais non idéale, dont l'existence n'est en rien un guide pour l’interprétation de la loi constitutionnelle.

Dans un élan de présomption, M. Sirota va même jusqu'à soutenir ce qui suit :

Then again, under prof. St-Hilaire's and Québec's interpretation, the judges of the Supreme Court itself, no matter what their previous affiliation, would not be eligible to be appointed to Québec's courts under s. 98. Québec's lawyer did his best to laugh this question away when it was put to him at the hearing at the Supreme Court and, when pressed, utterly failed to answer. I do not think that, had he been in that lawyer's place, prof. St-Hilaire would have succeeded either. ${ }^{78}$

J'ignore ce qu'il veut dire exactement par " to succeed ". S'il veut dire "remporter sa cause ", alors je le laisse sans moi faire dans le "réalisme juridique " imaginaire et prédire le résultat d'une plaidoirie de ma part qui n'aura jamais eu lieu. Si en revanche il veut dire "avoir quelque chose de sensé à répondre ", alors voici ma proposition. J'aurais répondu que la Cour suprême du Canada n'est pas une cour fédérale. En effet, la représentation du Québec à la CSC répond au développement de la même logique que celle qui préside à l'article 98 LC 1867 (et corrélativement l'article 97). Compte tenu du fait qu'il est d'autant compréhensible que le constituant de 1867 n'ait pas prévu le cas inusité d'un juge de la CSC qui redescendrait d'un ou deux échelons dans la hiérarchie judiciaire qu'une telle cour n'était pas encore créée mais la seule

78. Id. 
compétence fédérale de ce faire prévue, et compte tenu aussi de l'avis relatif à la nomination du juge Nadon, qui s'explique par la constitutionnalisation, en 1982, de la composition de la CSC, on se rendra compte qu'il était facile de ma part d'admettre qu'on fasse une exception au texte des articles 97 et 98 en faveur des juges de la CSC. Une telle admission était d'autant compréhensible que, alors que la CSC vient, en les couronnant, s'intégrer au système judiciaire de chacune des provinces, cela n'est pas du tout le cas des cours fédérales qui forment, de manière exceptionnelle au principe selon lequel le système judiciaire de chacune des provinces applique aussi bien le droit fédéral que le droit provincial, un système parallèle.

\section{$* * *$}

Reste à voir, comme l'a relevé encore une fois Léonid Sirota ${ }^{79}$, si, par le truchement de son nouveau Code de déontologie des avocats, le Barreau du Québec n'a pas lui-même renoncé à la protection de l'avis rendu par la Cour suprême dans l'affaire Nadon. En effet, parmi les charges de juge, le nouvel article 139.1 du Code tient pour incompatible avec l'exercice de la profession d'avocat la seule "fonction de juge suivant la Loi sur les tribunaux judiciaires (chapitre T-16) et de juge municipal à titre permanent et à temps complet " 80 , ce qui exclut notamment celle de juge d'une cour fédérale. À cette question, la réponse du Barreau fut la suivante, sur laquelle mon lecteur saura bien se faire son opinion :

La refonte du Code de déontologie des avocats, comme vous le savez sans doute, a été amorcée en 2010. Ce travail a été confié à un comité consultatif dédié et constitué d'avocats qui ont apporté bénévolement leur expertise et leur collaboration à la rédaction. Ainsi qu'il en va pour tout dossier de cette nature, la proposition finale a été ratifiée par le Conseil général du Barreau.

79. Leonid SiRotA, "What Happened? ", Double Aspect, 28 avril 2015, en ligne : https://doubleaspectblog.wordpress.com/2015/04/28/whathappened/; Leonid SirotA, "L'Affaire Nadon : R.I.P.? ", Double Aspect, 23 avril 2015, en ligne: https://doubleaspectblog.wordpress.com/ 2015/04/23/laffaire-nadon-r-i-p/.

80. $\quad$ Code de déontologie des avocats, R.L.R.Q., c. B-1, art. 139.1. 
Dans la rédaction des articles du Code visés par cette refonte, il importe de souligner que l'aspect politique n'a jamais été pris en considération. Seuls les principes éthiques et déontologiques ont balisé ce travail considérable.

En ce qui a trait à la formulation de l'article 139, alinéa 1, $\mathrm{du}$ Code de déontologie, nous avons effectué certaines vérifications sur les amendements proposés au cours des 4 années de refonte du Code qui ont mené à cette formulation finale.

Étant donné que nous souhaitons éviter l'interprétation que vous évoquez, le Barreau du Québec a demandé à l'Office des professions un amendement à cet article afin que celuici reflète mieux nos intentions. L'une des hypothèses envisagées pour cet amendement serait de laisser tomber la référence à la Loi sur les tribunaux judiciaires (chapitre T16) et d'inclure tous les juges fédéraux. ${ }^{81}$

81. Leonid SiRota, "The Barreau Responds ", Double Aspect, 30 avril 2015, en ligne : https://doubleaspectblog.wordpress.com/2015/04/30/thebarreau-responds/. 